risk to human health which can be directly used for informing decisions about how to manage those risks. Prerequisite of regulatory risk assessment is a clear causal association and exposureresponse-relationship. Risk quantification should be based on absolute measures instead of relative measures. Lifetime (excess) risk estimation is the most suitable effect measure in regulatory risk assessment. It provides the estimated probability of disease occurrence due to life long exposure to a special occupational hazard under certain exposure level. Methods on the quantification of Lifetime (excess) risk estimations are going to be introduced based on examples of silica related health issues, such as silicosis and lung cancer. Interpretation of risk estimations should be made carefully, especially regarding the biological plausibility of the estimated exposure-response-relationship, change of exposure patterns over working time, consideration of disease latency and comparability of exposure assessment methods used in various studies or countries.

\section{P1-346 EPIDEMIOLOGY AND INDIGENOUS HEALTH POLICY: RISING TO THE CHALLENGE}

doi:10.1136/jech.2011.142976f.38

R Supramaniam,* A Tang, D O'Connell. Cancer Council NSW, Sydney, New South Wales, Australia

Introduction The Aboriginal Patterns of Cancer Care Project (APOCC) is investigating why Aboriginal people with cancer in New South Wales (NSW) Australia have a 60\% higher mortality than non-Aboriginal people. Translating the results from APOCC to effect changes policy practice in an Indigenous health context presents a complex challenge.

Methods The APOCC team hosted a 1 day workshop bringing together policymakers, Aboriginal community members, health service providers, clinicians, health advocates and researchers to understand their needs and to leverage their expertise and networks of influence. We used several different methods to facilitate wider discussion about the dissemination and implementation of APOCC's findings.

Results Outcomes from APOCC will provide important evidence for decision making on delivering cancer services to NSW Aboriginal people. However to positively impact policymakers, communities and health service providers will require engaging a wide network of non-research, even non-health, organisations and individuals. The central principles of cultural safety, respect, reciprocity, consultation and relevance must overlay the presentation and implementation of APOCC's future recommendations in order to reduce the $60 \%$ higher mortality from cancer for Aboriginal people.

Conclusions Reliable epidemiological evidence is, and should, underpin shifts in public health policy. However translating results into policy and action to improve Aboriginal cancer mortality will require the efforts and cooperation of many individuals and organisations beyond the research community. This workshop has provided the researchers with invaluable initial insights and networks to address the complex challenges that will be faced in translating the findings into a reduction in cancer mortality for Aboriginal people.

\section{P1-347 PHYSICAL ACTIVITY AND ALL-CAUSE MORTALITY: FINDINGS FROM THE JAPAN COLLABORATIVE COHORT STUDY}

doi:10.1136/jech.2011.142976f.39

${ }^{1} \mathrm{~S}$ Suzuki, ${ }^{1} \mathrm{M}$ Kojima, ${ }^{1,2} \mathrm{~S}$ Tokudome, ${ }^{3} \mathrm{~K}$ Wakai, ${ }^{3} \mathrm{~T}$ Kondo, ${ }^{3} \mathrm{~K}$ Tamakoshi, ${ }^{4}$ A Tamakoshi. ${ }^{1}$ Nagoya City University, Nagoya, Japan; ${ }^{2}$ National Institute of Health and Nutrition, Tokyo, Japan; ${ }^{3}$ Nagoya University, Nagoya, Japan; ${ }^{4}$ Aichi Medical University, Toyoake, Aichi, Japan

Purpose To examine prospectively the association between physical activity and risk of all-cause death in Japanese population.
Methods We analysed data from the Japan Collaborative Cohort (JACC) Study, which included 70048 participants (29550 men and 40498 women), aged 40-79 years at baseline (1988-1990), who reported no previous history of cancer, and provided information on their walking and exercise habits. The subjects were followed prospectively from enrolment until 2003. Physical activity was evaluated using the time spent walking per day, and that spent exercising per week. The Cox proportional hazards model was used to estimate the HR for the association.

Results During the 1001870 person-years of follow-up, we identified 12051 deaths. Both among men and women, exercise was associated with lower mortality with a linear trend. The most physically active group (who walked for $\geq 1 \mathrm{~h}$ /day and exercised for $\geq 3 \mathrm{~h} /$ week) had a lower risk of death ( $\mathrm{HR}=0.67,95 \%$ CI 0.62 to 0.73 for men, and $\mathrm{HR}=0.75,95 \%$ CI 0.67 to 0.84 for women) compared with the least active group after adjusting for potential confounding factors. The results were not changed after excluding the deaths within first 2 years. The effect of physical activity on mortality was stronger among older subjects among men and women.

Conclusions Our analysis provided evidence that physical activity decreased the risk of all cause of death.

\section{P1-348 LEISURE-TIME PHYSICAL ACTIVITY AND BREAST CANCER RISK DEFINED BY OESTROGEN AND PROGESTERONE RECEPTOR STATUS: THE JAPAN PUBLIC HEALTH CENTER-BASED PROSPECTIVE STUDY}

doi:10.1136/jech.2011.142976f.40

${ }^{1} \mathrm{R}$ Suzuki, ${ }^{*} \mathrm{M}$ Iwasaki, ${ }^{2} \mathrm{~S}$ Yamamoto, ${ }^{1} \mathrm{M}$ Inoue, ${ }^{1} \mathrm{~S}$ Sasazuki, ${ }^{1} \mathrm{~N}$ Sawada, ${ }^{1} \mathrm{~T}$ Yamaji, ${ }^{1} \mathrm{~T}$ Shimazu, ${ }^{1} \mathrm{~S}$ Tsugane. ${ }^{1}$ Epidemiology and Prevention Division, Research Center for Cancer Prevention and Screening, National Cancer Center, Tsukiji, Chuo-ku, Tokyo, Japan; ${ }^{2}$ Cancer Information Services and Surveillance Division, Center for Cancer Control and Information Services, National Cancer Center, Tsukiji, Chuo-ku, Tokyo, Japan

Objective To investigate the association between leisure-time physical activity (LPA) and breast cancer risk in consideration of tumour oestrogen/progesterone-receptor (ER/PR) status.

Methods We conducted a population-based prospective cohort study among 53578 women in the Japan Public Health Center-based Prospective Study. LPA was assessed by self-reported questionnaires. A Cox proportional hazards regression model was used to derive RRs and $95 \%$ CIs.

Results From 1990 to 1993 to the end of 2007, 652 cases were identified. The absolute rate of breast cancer was 84 per 100000 person-years among the sedentary groups (?3 days/month). We observed a statistically significant inverse association between LPA and breast cancer risk (RR ?3 days/week vs ?3 days/month $=0.73$ $95 \%$ CI 0.54 to 1.00 ; p trend 0.037$)$, particularly in $E R+P R+(R R$ 0.43 ; $95 \%$ CI 0.19 to 1.00 ; ptrend 0.022 ) and this inverse trend was apparent among postmenopausal women (RR 0.25; 95\% CI 0.06 to 1.06; $p$ trend 0.041). An inverse trend was also observed between daily total physical activity and postmenopausal $\mathrm{ER}+\mathrm{PR}+$ risk $(p=0.046)$. Among BMI ? $25 \mathrm{~kg} / \mathrm{m} 2$ group, LPA was associated with decreased risk (RR ?1 days/week vs ?3 days/month $=0.65 ; 95 \% \mathrm{CI}$ 0.43 to 0.97 ; $p$ trend 0.033 ).

Conclusion Active participation in LPA may contribute to a decrease in breast cancer risk, particularly for postmenopausal ER+PR+ tumours. 